\title{
Clinical profile and difficulties in diagnosis of central nervous system infections in adult patients in a tertiary care hospital
}

\author{
U K Ranawaka ${ }^{1}$, E G D S Rajindrajith ${ }^{1}$, K V H K K Perera $^{1}$, K M M P Dassanayake ${ }^{2}$, B A H R Premaratne ${ }^{1}$, \\ H J de Silva ${ }^{1}$ \\ (Index words: central nervous system infections, meningitis, encephalitis, Sri Lanka, diagnosis)
}

\begin{abstract}
Objectives We aimed to describe the pattern of central nervous system (CNS) infections and accuracy of diagnosis in a Sri Lankan tertiary care hospital.

Methods We prospectively studied all adult patients with suspected CNS infection admitted over a two-year period. Data were collected on demographic and clinical features, laboratory findings, treatment and immediate outcome. Diagnosis of CNS infection was categorized as definite, probable, possible and uncertain.

Results We studied 215 patients [59.1\% males; mean age (SD) 44 (20) years]. Blood cultures were done in 65 (30.2\%) and only one was positive. Lumbar puncture was done in 146 (67.9\%), and cerebrospinal fluid Gram stains, culture and acid-fast bacilli stains were all negative. Diagnosis of CNS infection was considered 'definite' in only one patient, 'probable' in 57.2\%, 'possible' in 5.6\%, and 'uncertain' in 26\%. An alternative diagnosis was found in 23 patients (10.7\%). Intravenous antibiotics and aciclovir were given on emperical grounds, largely without microbiological confirmation.

Conclusions Diagnosis of CNS infections is highly unsatisfactory with available facilities, even in a tertiary care setting.
\end{abstract}

Ceylon Medical Journal 2013; 58: 26-28

\section{Introduction}

Central nervous system (CNS) infections are commoner and produce more deaths and disability in developing countries, but their management in these countries is difficult due to resource constraints. Clinical decisions on presumptive diagnosis and emperical treatment can be guided by knowledge of local disease patterns, but such epidemiological data are often lacking from developing countries. Previous published work from Sri Lanka is limited to case reports and few reports on specific situations. We aimed to describe the clinical spectrum, accuracy of diagnosis and management of CNS infections in unselected patients presenting to a Sri Lankan tertiary care hospital.

\section{Methods}

We conducted a prospective observational study of all patients admitted with presumed CNS infection to the adult medical wards of the Colombo North Teaching Hospital, Ragama over a two-year period. Patients were included based on any combination of the following: triad of fever, headache and vomiting, altered consciousness, seizures, focal neurological deficits, altered behaviour and meningism [1-3]. Data were collected by pre-intern medical officers after informed written consent. No additional interventions were done for the purpose of the study. Approval was granted by the Ethics Review Committee of the Faculty of Medicine, University of Kelaniya.

We collected data on demographic characteristics, clinical features, investigation findings, treatment and immediate outcome. The clinical syndrome of CNS infection was classified as meningitis (bacterial, tuberculous or viral), encephalitis, non-specific 'meningoencephalitis' (both meningitic and encephalitic features), cerebral malaria and cerebral abscess. Data were reviewed after discharge (or death) to assess the accuracy of diagnosis, and the proportion of patients in whom a definitive aetiological diagnosis was established. The final diagnosis of CNS infection was categorised as definite, probable, possible and uncertain, based on the strength of association between clinical features and investigation findings, modified from previously described criteria [1-3]. Patients in whom a diagnosis other than CNS infection was established were classified as 'alternate diagnosis'.

\section{Results}

We studied 215 patients [127 males (59.1\%); mean age (SD) 44 (20) years; age range 12-92 years]. The main clinical features and investigation findings are shown in the Table 1.

${ }^{1}$ Department of Medicine, Faculty of Medicine, University of Kelaniya and ${ }^{2}$ Colombo North Teaching Hospital, Ragama, Sri Lanka.

Correspondence: UKR, email: <udayaran@yahoo.com>. Received 3 August and revised version accepted 18 September 2012. Competing interests: none declared. 
Table 1. Main clinical features and investigation findings $(n=215)$

\begin{tabular}{|c|c|c|}
\hline Clinical Features & No. present & $\%$ \\
\hline Fever & 179 & 83.3 \\
\hline Altered consciousness & 149 & 69.3 \\
\hline Headache & 153 & 71.2 \\
\hline Vomiting & 116 & 54.0 \\
\hline Photophobia & 53 & 24.7 \\
\hline Behavioral changes & 101 & 47.0 \\
\hline Focal neurological signs & 33 & 15.3 \\
\hline Seizures & 59 & 27.4 \\
\hline Meningism & 92 & 42.8 \\
\hline Investigations & $\begin{array}{l}\text { No. present/ } \\
\text { no. performed }\end{array}$ & $\%$ \\
\hline Elevated ESR ( $>20 \mathrm{~mm} / 1 \mathrm{st} \mathrm{h})$ & $44 / 140$ & 31.4 \\
\hline Elevated CRP $(>12 \mathrm{mg} / \mathrm{L})$ & $38 / 54$ & 70.3 \\
\hline Elevated WBC count $\left(>11000 / \mathrm{mm}^{3}\right)$ & $124 / 204$ & 60.8 \\
\hline Positive blood culture & $1 / 65$ & 1.5 \\
\hline CSF analysis done & 146 & $7.9 *$ \\
\hline elevated protein $(>45 \mathrm{mg} / \mathrm{dl})$ & $100 / 146$ & 68.5 \\
\hline low glucose $(<1 / 2$ RBS $)$ & $28 / 146$ & 19.2 \\
\hline $\mathrm{PMN}>0 / \mathrm{mm}^{3}$ & $64 / 146$ & 43.8 \\
\hline lymphocytes $>5 / \mathrm{mm} 3$ & $72 / 146$ & 49.3 \\
\hline positive Gram stain & 0 & - \\
\hline positive culture & 0 & - \\
\hline positive AFB stain & 0 & - \\
\hline CT scan done & 151 & $0.2 *$ \\
\hline changes suggestive of CNS infection & $63 / 151$ & 41.7 \\
\hline EEG done & 83 & $8.6^{*}$ \\
\hline changes suggestive of CNS infection & $19 / 83$ & 22.9 \\
\hline
\end{tabular}

* Percentages are derived from the total number of patients (215) RBS - random blood sugar; PMN - polymorphonuclear leucocytes; AFB - acid-fast bacilli

Thirty-three patients (32\% out of 103 where information was available) had received antibiotics before admission. Blood cultures were done in 65 patients (30.2\%), and only one was positive (Streptococcus pneumoniae). Lumbar puncture was done in 146 (67.9\%). CSF Gram stain, culture and staining for acid-fast bacilli (AFB) were all negative. Blood films were checked for malarial parasites in 26 clinically suspected patients, and all were negative.

A 'definite' diagnosis with microbiological confirmation was made only in one patient. Diagnosis of CNS infection was considered 'probable' in 123 patients (57.2\%) and 'possible' in 12 (5.6\%). Of these categories ('definite', 'probable' or 'possible'), the syndromic diagnosis was meningitis in 36 patients (16.7\%), encephalitis in $33(15.3 \%)$ and a non-specific meningo-encephalitis in 63 (29.3\%). Four patients had a cerebral abscess. No cases of CNS tuberculosis or cerebral malaria were positively identified. Diagnosis of CNS infection was considered 'uncertain' in 56 patients $(26 \%)$, and an 'alternative diagnosis' was found in 23 patients $(10.7 \%)$.

All patients were given antimicrobial therapy on emperical grounds, without microbiological confirmation (except in one). One hundred and eighty one patients $(84.2 \%)$ were treated with intravenous cephalosporins, and $133(61.9 \%)$ with intravenous aciclovir. Intravenous steroids were used in 82 patients (38.1\%). Twenty patients $(9.3 \%)$ died during hospital stay.

\section{Discussion}

In this prospective observational study of all patients with suspected CNS infection admitted to a teaching hospital over a two-year period, a definitive aetiological diagnosis was possible in only one patient. In about twothirds $(62 \%)$ of patients where a diagnosis of CNS infection seemed highly likely ('probable'and 'possible' categories), we could not establish a microbiological causation.

Several factors are likely to have contributed to the low rate of positive diagnosis. Newer diagnostic techniques (bacterial and viral genome detection by PCR, bacterial antigen assays, automated culture systems) are not routinely available in the public sector hospitals in Sri Lanka. Some of the available routine investigations were not performed in all patients. Other factors that may contribute (delays in lumbar puncture and blood cultures, improper specimen handling, etc) were not analysed in the study.

Low isolation rates are common in developing countries, and lack of diagnostic facilities is considered to be the main reason for this [4,5]. However, low detection rates are commonly reported from these countries even in collaborative studies conducted with good microbiological support [6-8]. Prior antibiotic use is common (27-53\%) and likely to be an important contributory factor, similar to our study $[5,7,9]$.

Comparisons of detection rates between studies using different methodologies can be misleading. Most studies with high isolation rates have described data from one selected syndrome (meningitis, encephalitis, etc.) or included only microbiologically proven cases [10-12]. Data from unselected patients recruited with clinically suspected CNS infection, as in our study, are limited, and show much lower rates of isolation even from developed countries [1-3]. In a multicentre study from UK, only $20 \%$ of suspected patients were found to have a CNS infection, and CSF culture was positive in only 3 out of 199 samples [1]. In the California Encephalitis Project, an aetiological agent was not found in $62 \%$ of patients despite extensive 
testing [2]. Up to $85 \%$ of cases worldwide are reported to be due to undetected causes [3]. Several factors, such as a wider spectrum of neurotropic organisms not detected by routine testing, and non-infectious causes of encephalitis (paraneoplastic, immune-mediated), can contribute to the low diagnostic yield [3].

This is the first prospective observational study of CNS infections reported from Sri Lanka. We were unable to detect an aetiological agent in most of our patients, and many were treated with antimicrobials on emperical grounds. Improved diagnostic facilities in public sector hospitals is likely to minimise treatment costs, in addition to improving patient care.

\section{Acknowledgements}

We thank the following members of the Ragama C N S Infection Study Group: A N H M U K G D B Nawaratne, M L Harshani, V N R M Fonseka, R L P Harshani, K L W Hathagoda, W A L K Weerasooriya (all of Faculty of Medicine, University of Kelaniya); A Kulatunga, W A J N Tissera, M M S Cooray, K Thirumavalavan, G Premawansa, S M S B Samarakoon (all from Colombo North Teaching Hospital, Ragama).

\section{References}

1. Michael BD, Sidhu M, Stoeter D, et al. North West Neurological Infections Network. Acute central nervous system infections in adults - a retrospective cohort study in the NHS North West region. Quarterly Journal of Medicine 2010; 103: 749-58.

2. Glaser CA, Gilliam S, Schnurr D, et al. In search of encephalitis etiologies: diagnostic challenges in the California Encephalitis Project, 1998-2000. Clinical Infectious Diseases 2003; 36: 731-42.

3. Granerod J, Ambrose HE, Davies NW, et al. UK Health Protection Agency (HPA) Aetiology of Encephalitis Study Group. Causes of encephalitis and differences in their clinical presentations in England: a multicentre, population-based prospective study. Lancet Infectious Diseases 2010; 10: $835-44$.

4. Scarborough M, Thwaites GE. The diagnosis and management of acute bacterial meningitis in resource-poor settings. Lancet Neurology 2008; 7: 637-48.

5. Sacchi CT, Fukasawa LO, Gonçalves MG, et al. Incorporation of real-time PCR into routine public health surveillance of culture negative bacterial meningitis in São Paulo, Brazil. PLoS One 2011; 6: e20675.

6. Batuwanthudawe R, Karunarathne K, Dassanayake M, et al . Surveillance of invasive pneumococcal disease in Colombo, Sri Lanka. Clinical Infectious Diseases 2009; 48 (Sup 2): S136-40.

7. Shah AS, Knoll MD, Sharma PR, et al. Invasive pneumococcal disease in Kanti Children's Hospital, Nepal, as observed by the South Asian Pneumococcal Alliance network. Clinical Infectious Diseases 2009; 48 (Sup 2): S123-8.

8. Le VT, Phan TQ, Do QH, et al. Viral etiology of encephalitis in children in southern Vietnam: results of a one-year prospective descriptive study. PLoS Neglected Tropical Diseases 2010; 4: e854.

9. Zaidi AK, Khan H, Lasi R, Mahesar W. Sindh Meningitis Group. Surveillance of pneumococcal meningitis among children in Sindh, southern Pakistan. Clinical Infectious Diseases 2009; 48 (Sup 2): S129-35.

10. Weisfelt M, van de Beek D, Spanjaard L, et al. Clinical features, complications, and outcome in adults with pneumococcal meningitis: a prospective case series. Lancet Neurology 2006; 5: 123-9.

11. Aronin SI, Peduzzi P, Quagliarello VJ. Community-acquired bacterial meningitis: risk stratification for adverse clinical outcome and effect of antibiotic timing. Annals of Internal Medicine 1998; 129: 862-69.

12. van de Beek D, de Gans J, Spanjaard L, et al. Clinical features and prognostic factors in adults with bacterial meningitis. New England Journal of Medicine 2004; 351: 1849-59. 